\title{
The impact of loneliness and social isolation on health state utility values: a systematic literature review
}

\author{
Ishani K. Majmudar ${ }^{1}\left(\mathbb{D} \cdot\right.$ Cathrine Mihalopoulos $^{1} \cdot$ Bianca Brijnath $^{2} \cdot$ Michelle H. Lim $^{3} \cdot$ Natasha Yvonne Hall ${ }^{1}$. \\ Lidia Engel ${ }^{1}$
}

Accepted: 6 December 2021 / Published online: 24 January 2022

(C) The Author(s) 2021

\begin{abstract}
Background Loneliness and social isolation are recognised as social problems and denote a significant health burden. The aim of this study was to conduct a systematic literature review to explore the health state utility values (HSUVs) associated with loneliness and/or social isolation.

Method Peer-reviewed journals published in English language that reported both HSUVs along with loneliness and/or social isolation scores were identified through five databases. No restrictions were made relating to the population, study design or utility estimation method used.

Results In total, 19 papers were included; 12 included a measure of loneliness, four studies included a measure of social isolation and three studies considered both loneliness and social isolation. All studies focused on individuals with pre-existing health conditions-where the EQ-5D-3L instrument was most frequently used to assess HSUVs. HSUVs ranged from 0.5 to 0.95 in those who reported not being lonely, 0.42 to 0.97 in those who experienced some level of loneliness, 0.3 to 0.87 in those who were socially isolated and 0.63 to 0.94 in those who were not socially isolated.

Conclusion There was significant variation in HSUVs complicated by the presence of co-morbidities, population heterogeneity, variations in methods used to derive utility scores and differences in the measurement of loneliness and/or social isolation. Nevertheless, the lower HSUVs observed should be considered to significantly impact quality of life, though we also note the need for further research to explore the unique impact of loneliness and social isolation on HSUVs that can be used in the future economic evaluations.
\end{abstract}

Keywords Loneliness $\cdot$ Social isolation $\cdot$ Health state utility values $\cdot$ Economic burden $\cdot$ Health burden

\section{Background}

Loneliness and social isolation represent global public health concerns $[1,2]$. While the terms are often used interchangeably, they are not synonymous; social isolation is an objective state and increased social isolation can be quantified

Ishani K. Majmudar

Ishani.majmudar@deakin.edu.au

1 Faculty of Health, School of Health and Social Development, Institute for Health Transformation, Deakin Health Economics, Deakin University, Geelong, VIC, Australia

2 Social Gerontology, National Ageing Research Institute (NARI), Parkville, Australia

3 Centre for Mental Health, Swinburne University of Technology, Hawthorn, Australia by the reduced size of social networks and lack of social contact. Loneliness, also referred to as 'perceived social isolation', on the other hand, is a subjective experience, which occurs when a person feels a discrepancy between desired and actual social relationships $[3,4]$. Thus, social isolation is different from loneliness as one can have social connections and still feel lonely, or can be alone and may not feel lonely [5].

While every individual will experience loneliness at some point in their life, some age groups are more prone to loneliness, for example, late adolescence or older people [6]. Critical transitions during these life stages, from adolescence to adulthood, as well as decreasing economic and social resources, limitations in mobility and loss of spouse and relatives that are common in later life, are thought to explain why these age groups experience more loneliness and social isolation [6, 7]. Previous literature 
has demonstrated a link between loneliness and social isolation with increased risk of developing cardiovascular diseases [8], cognitive deterioration [9], increased blood pressure [10], infectious illnesses [11] and early mortality [12]. Loneliness and social isolation are also associated with increased risk of dementia [13], depression and suicide [14]. Moreover, it has been argued that the health impact of loneliness and social isolation can be worse than risk factors such as smoking or obesity [15]. It has also been found that older adults who experience "extreme loneliness" have a greater chance of premature death [16].

Ongoing strategies to tackle loneliness and social isolation through interventions have been developed over years [17-19]. Many of these interventions targeted older adults and reported some level of success in reducing loneliness and/or social isolation with factors such as adaptability, community development approach and productive engagement being associated with the most effective interventions [20]. However, whether such interventions provide 'good value for money' has largely remained unanswered. Given the scarcity of healthcare resources, it is important to identify not only effective interventions and programmes but also cost-effective strategies [19]. The most common type of economic evaluation is cost-utility analysis (CUA), which expresses results in terms of cost per quality-adjusted life year (QALY) gained [21]. To generate QALYs, length of life is adjusted by levels of healthrelated quality of life (HRQoL) using a single value known as health state utility value (HSUV). HSUV is measured on a scale of $0-1$, where 0 is equivalent to being dead and 1 is considered as perfect health [21]. While HSUV can be obtained using direct techniques (i.e. time trade-off, standard gamble or visual analogue scale), these weights are commonly derived indirectly through the use of questionnaires, referred to as multi-attribute utility instruments (MAUIs) or preference-based HRQoL instruments. These instruments consist of a descriptive system, where a set of items (or questions) elicits responses to the main dimensions of HRQoL measured by the questionnaire. A second component of MAUIs is the valuation system. MAUIs have utility formula attached to each response on an item/ domain, which returns a weight anchored between 0 and 1 for each set of responses. This formula reflects societal preferences that indicate the relative importance of each item and in turn each HRQoL domain to derive a single HSUV [21]. These values are used to compare the preferences of the general population for different health states across various diseases [22]. Therefore, MAUIs allow an intervention targeted to reduce loneliness to be compared with any other health condition. HSUVs and QALYs are commonly used outcome measures across all areas of economic evaluation and Health technology assessment agencies across the world.
The EQ-5D(-3L or -5L) [23], Health Utilities Index Mark 2 or Mark 3 (HUI2 and HUI3), Short-Form-6 Dimension (SF-6D)-derived from the SF12 or 36, quality of wellbeing (QWB) scale, the assessment of quality of life (AQoL) suite of instruments and 15 Dimension (15D) are the most popular MAUIs [24]. They differ in their conceptualisation, content, length and methods used for converting health state descriptions into utilities. No single instrument is considered a gold standard for a certain health state, although some countries have expressed a preference for a particular MAUI in their national guidelines (e.g. the United Kingdom prefers the EQ-5D-3L) [22]. The EQ-5D, HUI, QWB and 15D have items predominantly relating to physical health. The SF-6D has an equal number of items covering both physical and psychological domains, while the AQoL-8D has been developed to capture particularly psychosocial domains of HRQoL, including social functioning [24]. Using a suitable utility method and instrument can affect the estimated HSUVs and in turn influence cost-effectiveness results as different MAUIs produce different HSUVs even in the same people [25]. This is largely because the different MAUIs measure different HRQoL domains.

Economic evaluations can be conducted alongside clinical trials or via modelling techniques. Appropriate HSUVs are assigned to health states defined within a model for economic evaluation that individuals experience over time through their treatment pathway. HSUVs associated with these health states thus provide important inputs to conduct cost-effectiveness analysis. As it is practically not feasible for health economists to elicit individual HSUVs directly or indirectly for every study, reviews of HSUVs for use in individuals across health states can provide a readily available source of HSUVs that can be used in an modelled economic evaluation [25-27]. These reviews provide results that improve robustness, transparency and rigour of the economic model, allowing appropriate and systematic selection of model parameters as well as greater understanding of the 'burden of disease' for a particular health state. Although, several studies have previously conducted reviews to explore the health and economic burden of different health conditions [26, 28, 29], to the best of our knowledge, no published review has examined HSUVS by the presence/absence or the degree of loneliness and social isolation.

We aimed to systematically review the literature to assess the availability of HSUVs associated with loneliness and/or social isolation across all age groups, with the intention that the findings can inform future model-based economic evaluations as well as provide an indication of the burden potentially associated with loneliness and social isolation. This review will help demonstrate which measurement techniques were used to assess HSUVs, to inform future trial-based economic evaluation and provide information on the corresponding value of loneliness and/ or social isolation scores. 


\section{Methods}

A comprehensive systematic literature review was conducted according to the preferred reporting items for systematic reviews and meta-analysis (PRISMA) [30]. This review protocol was registered on the Prospero database [CRD42021243375].

\section{Study selection}

The search was conducted in November 2019 and updated in April 2021. The five databases searched included Medline Complete, PsycINFO, Embase, CINAHL and EconLit. A keyword search was performed using the terms related to concepts of loneliness, social isolation, measures of loneliness and measures of HSUVs. Search terms for each concept were combined along with Medical Subject Headings (MeSH) terms (Supplementary 1). Initial title abstract screening was conducted by two reviewers IM and NH using the Rayyan QCRI Tool [31]. A third author LE resolved disagreements. Full-text screening of the agreed articles from the first step was conducted by two authors with any discrepancies solved through discussion with the third author. Third, a backward citation search, which involved screening the titles and abstracts of the reference list of the included studies, was conducted using Scopus. All types of studies (observational and experimental designs) that have used direct elicitation methods or indirect methods to measure HSUVs and have corresponding loneliness/social isolation scores were included. The inclusion and exclusion criteria are stated in Table 1.

\section{Data extraction}

Data extraction was conducted by the lead author and checked by another author. To guide data extraction, predesigned tables were used. Adjustments to the tables were made to accommodate the information provided by the included articles.

The data extracted included the following:

(i) Descriptive data about lead author, publication year, country, study design and sample size;

(ii) Demographic data about target population, including presence of health conditions;

(iii) Utility valuation data: Utility score methods with reported mean utility value;

(iv) Loneliness and social isolation measure data: Measure of reporting loneliness and/or social isolation with the corresponding mean score.

We aimed to include all studies which reported a HSUV score alongside a loneliness/social isolation score, not limiting to studies that only included study participants who experienced loneliness and/or social isolation. HSUV scores were extracted either for the entire study sample along with a corresponding score from the loneliness measure and/or social isolation measure, or where available, by levels of loneliness and social isolation using instrument-specific cut-off points. Where the allocation of HSUVs by levels of loneliness and/or social isolation was not provided by authors, a categorisation of scores was made by the lead author based on the reported values and the cut-off points of the loneliness/social isolation measures used in the study by Zhu et al. [32]. Baselines scores were considered in studies which reported HSUVs at different time points.

\section{Results}

After deduplication, our search yielded 4590 unique references. After screening titles and abstracts, we reviewed 238 full texts, of which 16 studies met inclusion criteria. An updated search was undertaken, which further identified

Table 1 Inclusion criteria and exclusion criteria

\begin{tabular}{lll}
\hline & Inclusion criteria & Exclusion criteria \\
\hline $\begin{array}{l}\text { Population } \\
\text { Outcome of interest }\end{array}$ & All populations and age groups & Loneliness and/or social isolation \\
Type of study & Quantitative studies (cross-sectional, longitudinal, cohort, etc.), reported the & Qualitative studies \\
& scores of utility weights relating to loneliness or social isolation & Protocol papers, conference abstracts, \\
Utility instrument & Both direct elicitation methods and indirect utility valuation methods & reviews opinion and editori- \\
Country & All countries & als \\
Publication type & Published in peer-reviewed journals & Other Language \\
& &
\end{tabular}


three studies. The PRISMA flow diagram, presented in Fig. 1, shows the selection process with reasons of exclusion.

\section{Study characteristics}

There has been an increasing interest in the research area of loneliness and health outcomes over the last decade, as most of the included studies were published after 2010. Sample size varied from 20 to 2713 individuals in the reviewed studies. Included articles consisted of studies from Australia $(n=4)$ [33-36], UK $(n=3)$ [37-39], Singapore $(n=2)$ [40, 41], Netherlands $(n=2)[42,43]$, Canada and USA $(n=2)$ [44, 45], with one study from the Republic of Korea [46], China [32], Spain [47], Finland [48], Sweden [49] and Malaysia [50]. Thirteen studies included an older population [32, 34, 36, 38-40, 42, 44, 46-50] five studies included general adults [35, 37, 41, 43, 45] and one study focused solely on young adults aged 18-25 years [33]. There were nine cross-sectional studies [32, 38, 41, 44-47, 49, 50], eight interventional studies [34, 36, 37, 39, 40, 42, 43, 48], one

Fig. 1 PRISMA Diagram

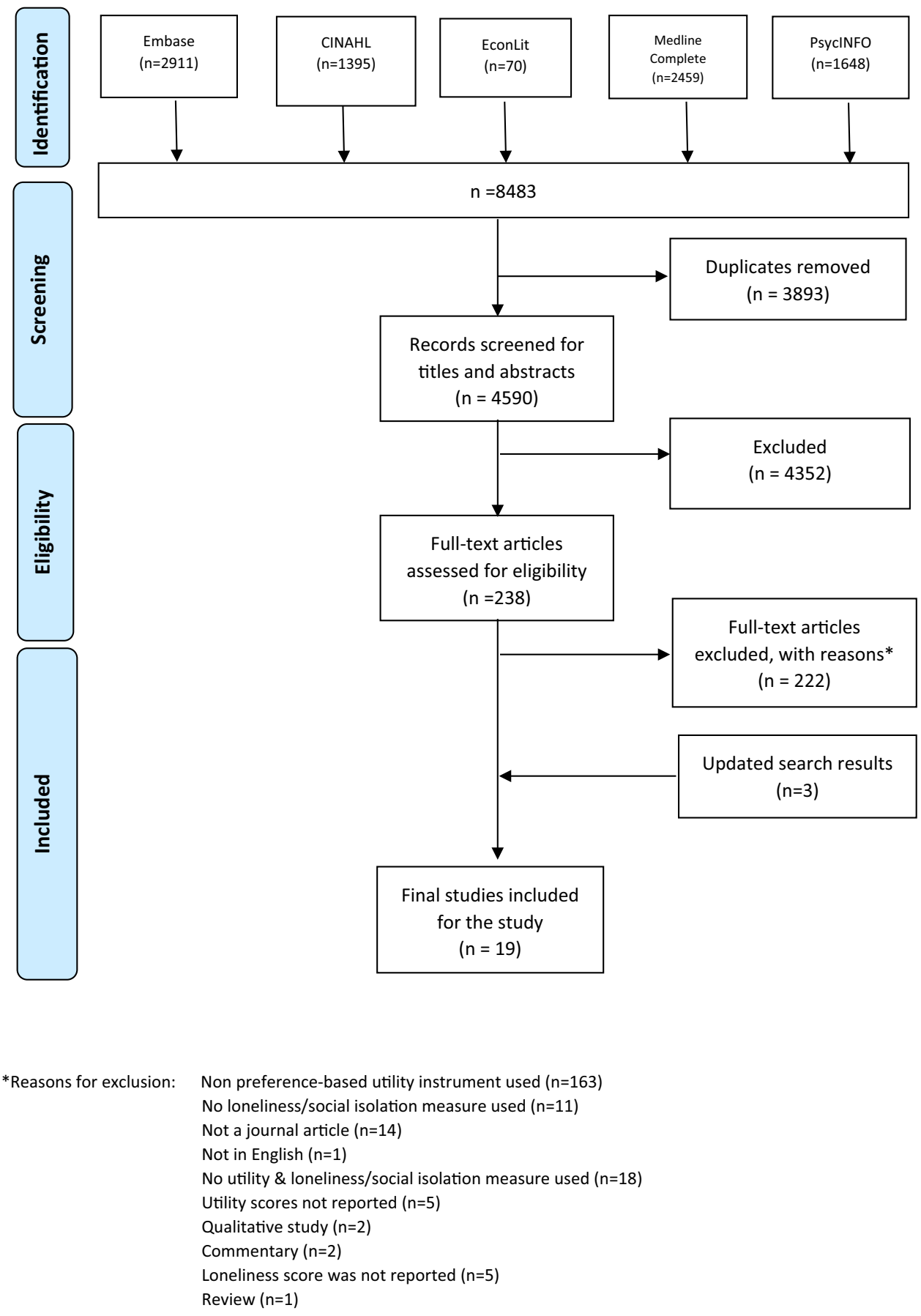


observational study [33] and one quasi-experimental study [35]. Ten studies included community-dwelling participants $[32,37-42,44,48,49]$, two studies focused on institutionalised vs non-institutionalised older adults, while the remaining studies did not clearly specify the living situation of study participants $[45,47,50]$. Two studies recruited participants specifically experiencing loneliness $[43,48]$ while most studies included participants with various other health conditions, such as sight loss [37], hearing loss [36], serious mental illness [33, 50], depression [46], skin disease [41], HIV [45] and multiple co-morbidities [34, 35, 40, 42, 44, 47, 48].

HSUVs were reported in twelve studies alongside a measurement of loneliness, four studies alongside a measure of social isolation and three studies considered both. There were inconsistencies in the way loneliness and social isolation were reported and very few studies categorised the study sample into degrees of loneliness and/or social isolation [32, 38]. Table 2 describes the overview of instruments used. Further characteristics of studies that reported HSUVs by level of loneliness and social isolation are presented in Table 3 and 4, respectively.

\section{Studies measuring Loneliness}

Studies that included a loneliness measure valued health states using various MAUIs, including the EQ-5D-3L [32, 37, 39, 41-43, 45-47, 49], AQoL-8D [33, 35], 15D [48], HUI2 [44] and HUI3 [36]. Measures of loneliness included the UCLA 20-item scale [32, 37, 46], R-UCLA 20-item [33], R-UCLA 3-item [35, 41], 11-item De Jong Gierveld Scale [36, 39, 42, 43], 6-item De Jong Gierveld Scale [47] and study specific questions asking individuals directly whether they are lonely or not $[35,44,45,48,49]$.

Figure 2 demonstrates the reported HSUVs using various MAUIs by the reported degree of loneliness. The HSUVs of the overall sample included in the studies ranged from as low as 0.42 to as high as 0.95 . Those identified as 'being lonely' or 'not lonely' by the respective study or using the categorisation based on cut-off points of the loneliness-specific measures, reported HSUVs ranging from 0.46 to 0.81 for the 'lonely' sample, whereas scores ranged from 0.5 to 0.95 for those who reported 'not being lonely'. Only one study reported HSUVs by the degree of loneliness using the EQ5D-3L (HSUVs: low $=0.97$, moderate $=0.93$, moderately high $=0.86$ ) [32]. This study demonstrated a significant difference among the HSUVs across the levels of loneliness. Remaining studies were categorised by levels of loneliness using cut-off points from Zhu et al. [32]. HSUVs for 'low level of loneliness' reported by one study was 0.97 [32], whereas HSUVs for 'moderate level of loneliness' and 'moderately high' ranged from 0.65 to 0.93 and from 0.42 to 0.86 [32], respectively. When using the AQoL-8D, HUI2 and HUI3, lower HSUVs were reported compared with scores using the EQ-5D-3L.

The included studies consisted of participants with varying population characteristics and health conditions. Two studies focused specifically on participants having a subjective feeling of loneliness, which reported a HSUV of 0.46 [43] and 0.78 [48]. Two studies, which included a population with serious mental health problems, such as depressive disorders, anxiety disorders, schizophrenia, personality disorder and others, reported HSUV of 0.81 associated with moderate levels of loneliness [46] and 0.42 with moderately high levels of loneliness [33]. While most studies had a mean age greater than 50 years, one study considered younger adults with serious mental illness and reported that high levels of loneliness were associated with a HSUV of 0.42 [33]. A study which explored HSUVs among people with chronic diseases reported 'moderately lonely' scores with HSUVs ranging from 0.62 to 0.72 [42]. Two studies, including participants with chronic health conditions, reported HSUVs for

Table 2 Overview of instruments used

\begin{tabular}{|c|c|c|c|c|c|}
\hline \multicolumn{2}{|l|}{ Utility } & \multicolumn{2}{|l|}{ Loneliness } & \multicolumn{2}{|l|}{ Social isolation } \\
\hline Measure & \# & Measure & $\#$ & Measure & \# \\
\hline EQ-5D (-3L/-5L) & $\begin{array}{l}12[32,34,37, \\
39-43,45-47, \\
49]\end{array}$ & UCLA LS 20 item & $3[32,37,46]$ & Lubben Social Network Scale-revised & $1[36]$ \\
\hline AQoL-8D & $3[33,35,50]$ & $\begin{array}{l}\text { Six-item De Jong } \\
\text { Gierveld Loneliness } \\
\text { Scale }\end{array}$ & $1[47]$ & The Lubben Social Network Scale- 6 item & $2[40,41]$ \\
\hline HUI2 & $1[44]$ & $\begin{array}{l}\text { De Jong Gierveld } \\
\text { Loneliness Scale } \\
\text { 11-item scale }\end{array}$ & $4[36,39,42,43]$ & Friendship Scale & $2[34,50]$ \\
\hline HUI3 & $1[36]$ & R-UCLA LS 3 item & $2[35,41]$ & Social inclusion & $1[33]$ \\
\hline $15 \mathrm{D}$ & $1[48]$ & R-UCLA LS 20 item & $1[33]$ & Social health battery & $1[38]$ \\
\hline EQ-5D + SF-6D & $1[38]$ & Single question & $5[35,44,45,48,49]$ & & \\
\hline
\end{tabular}




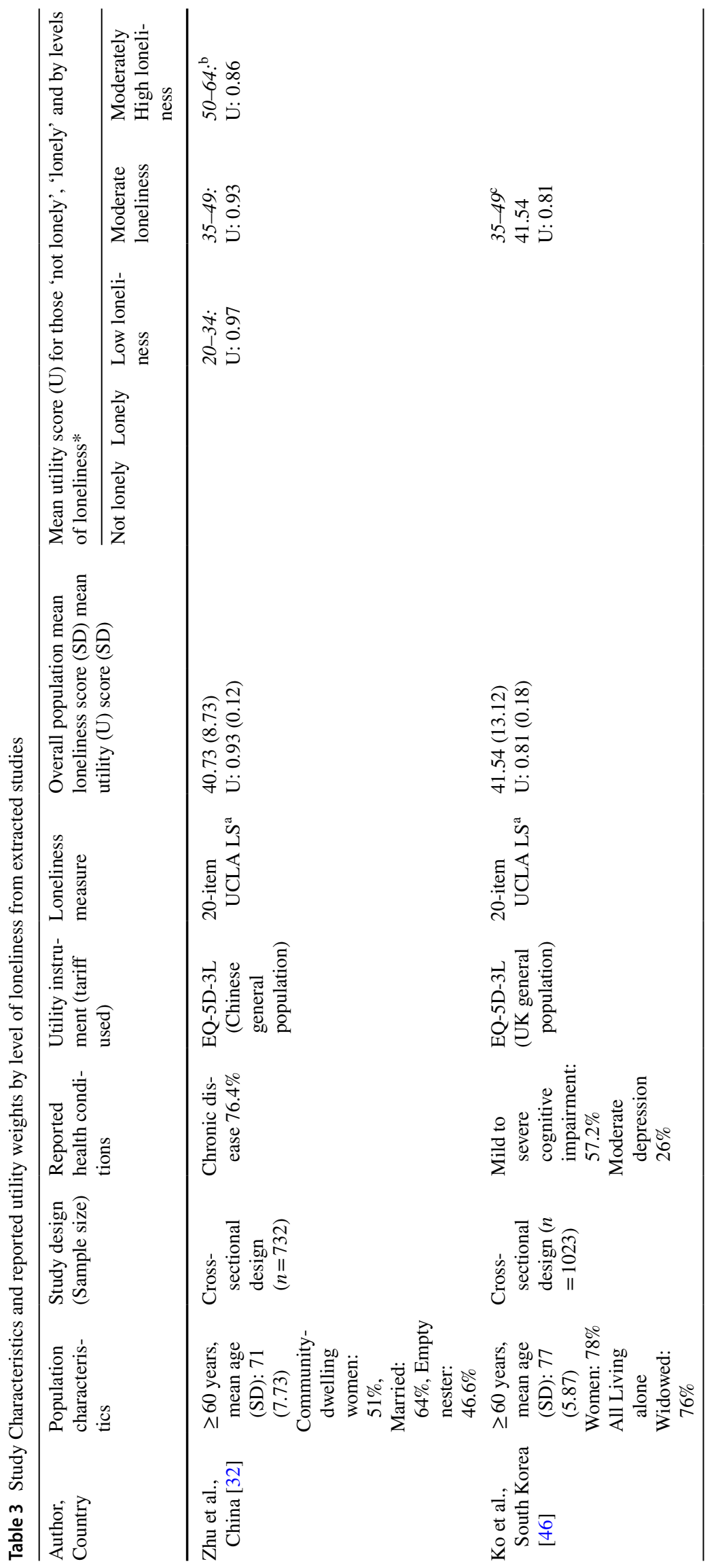




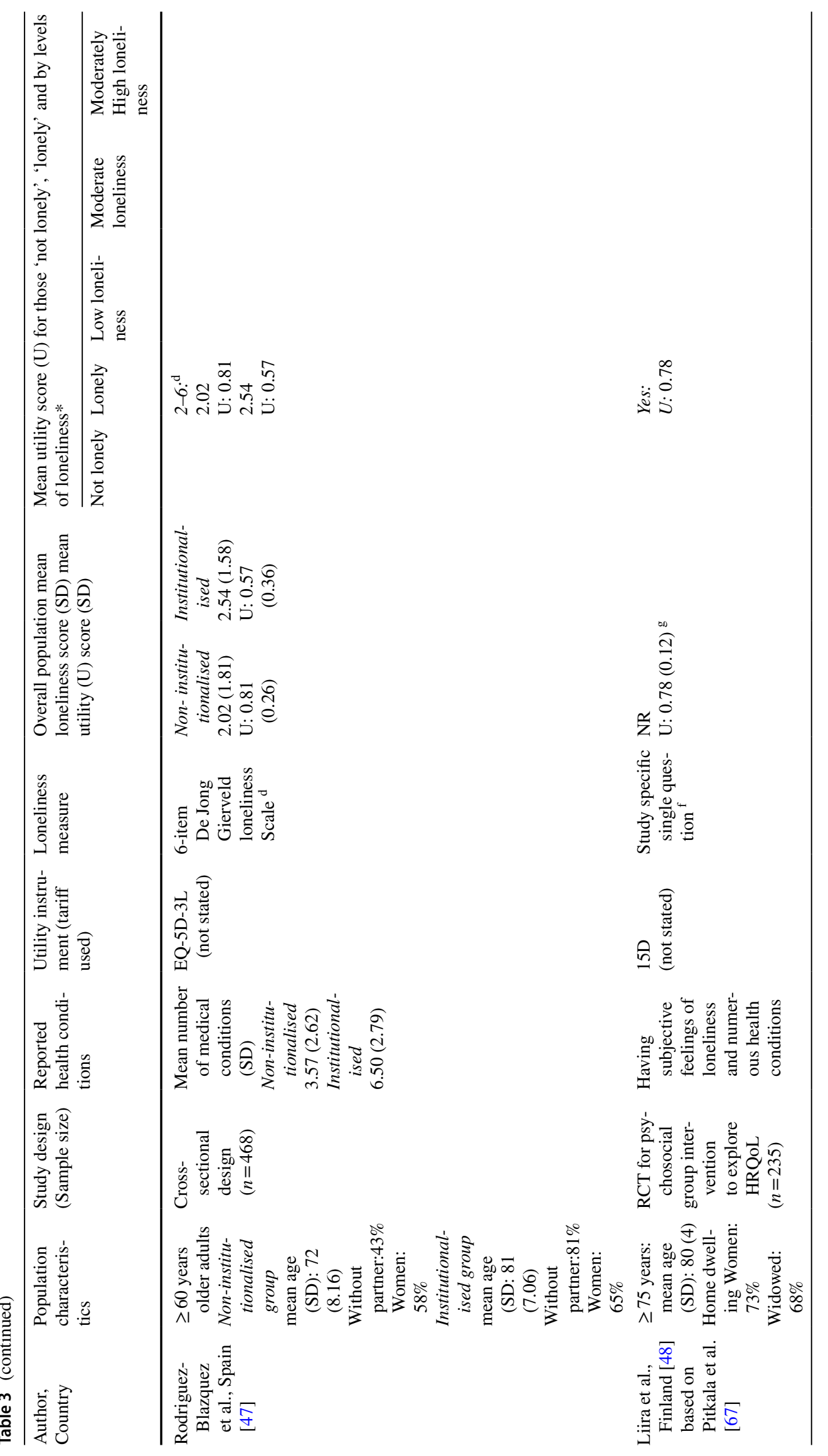




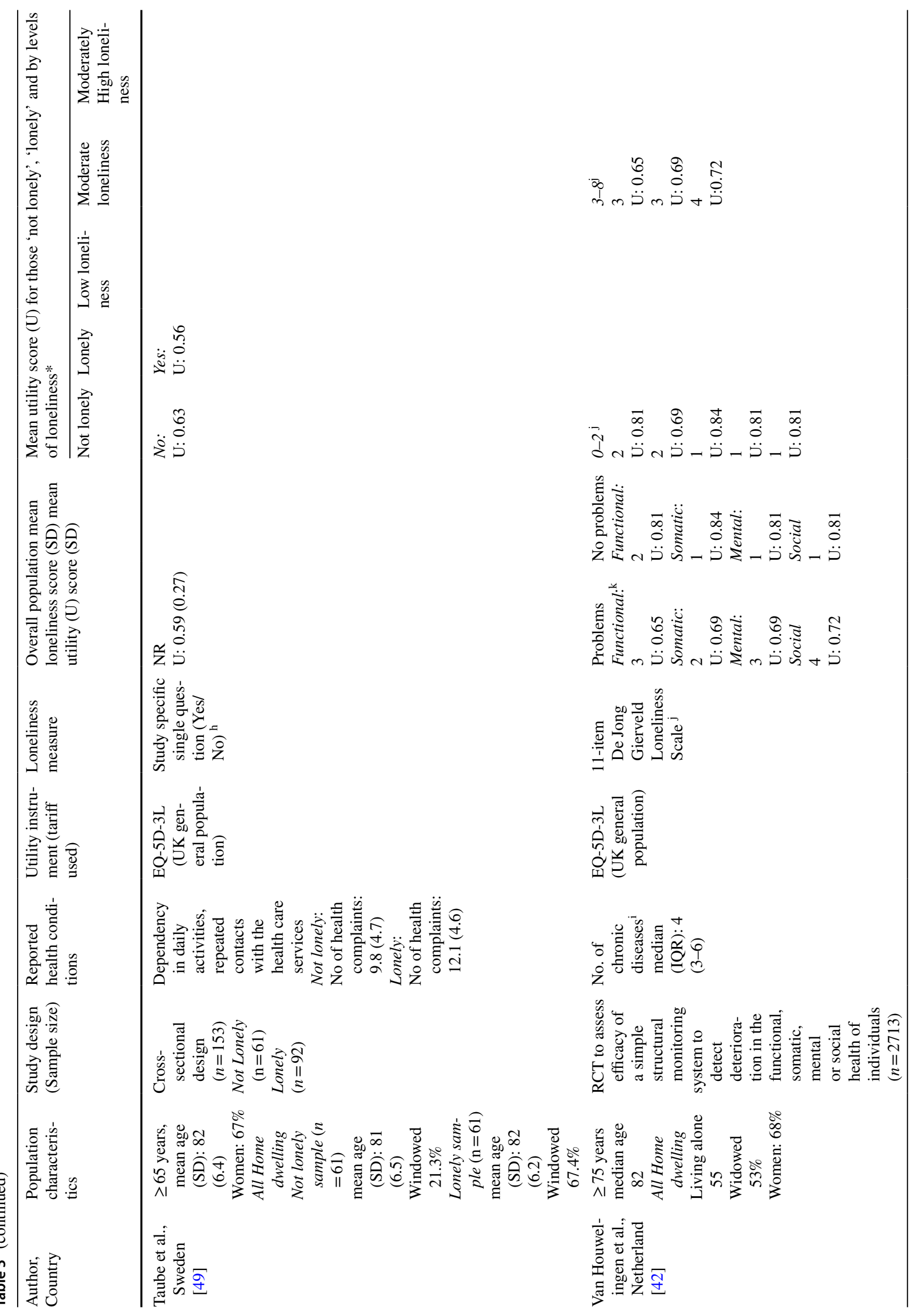




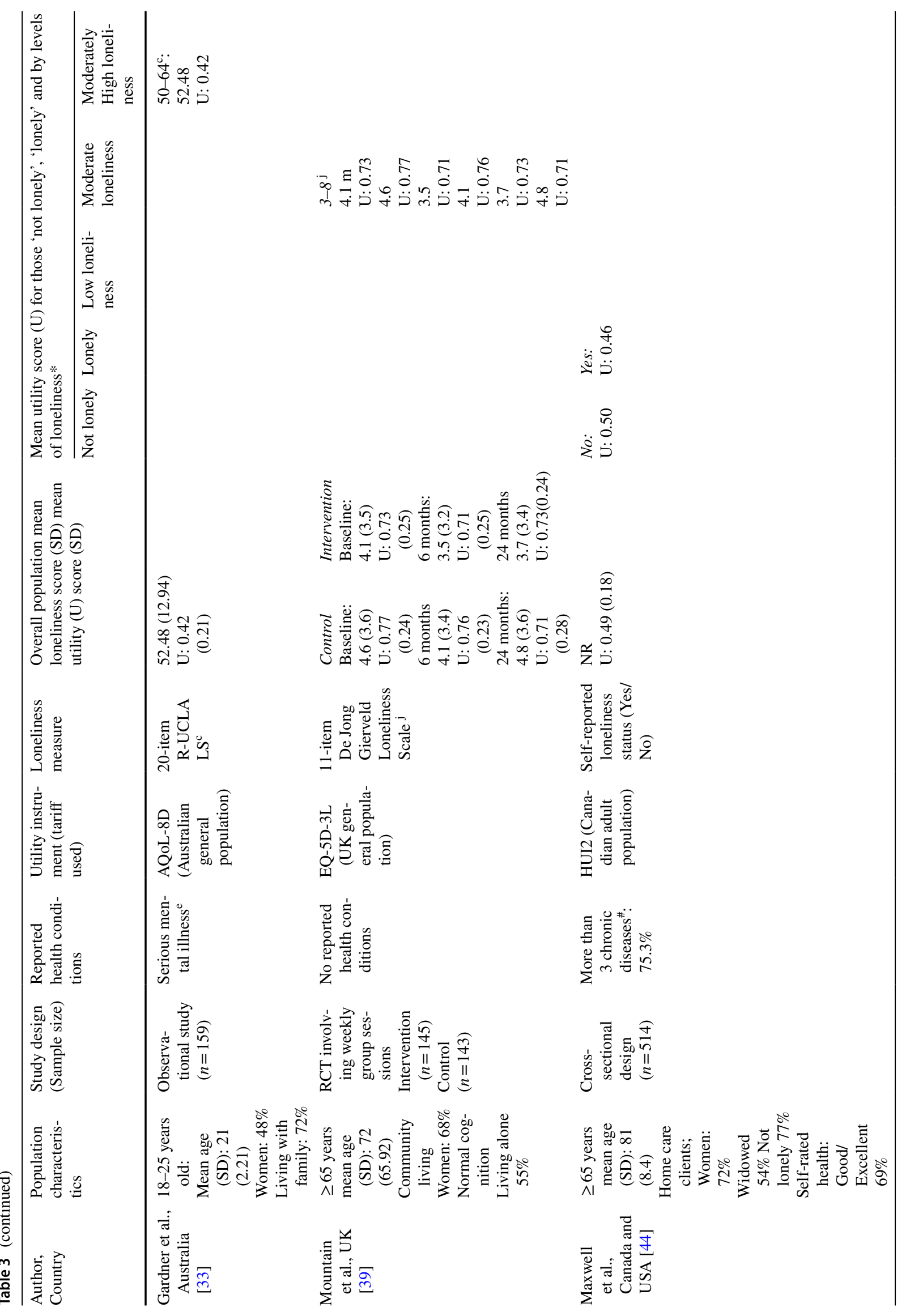




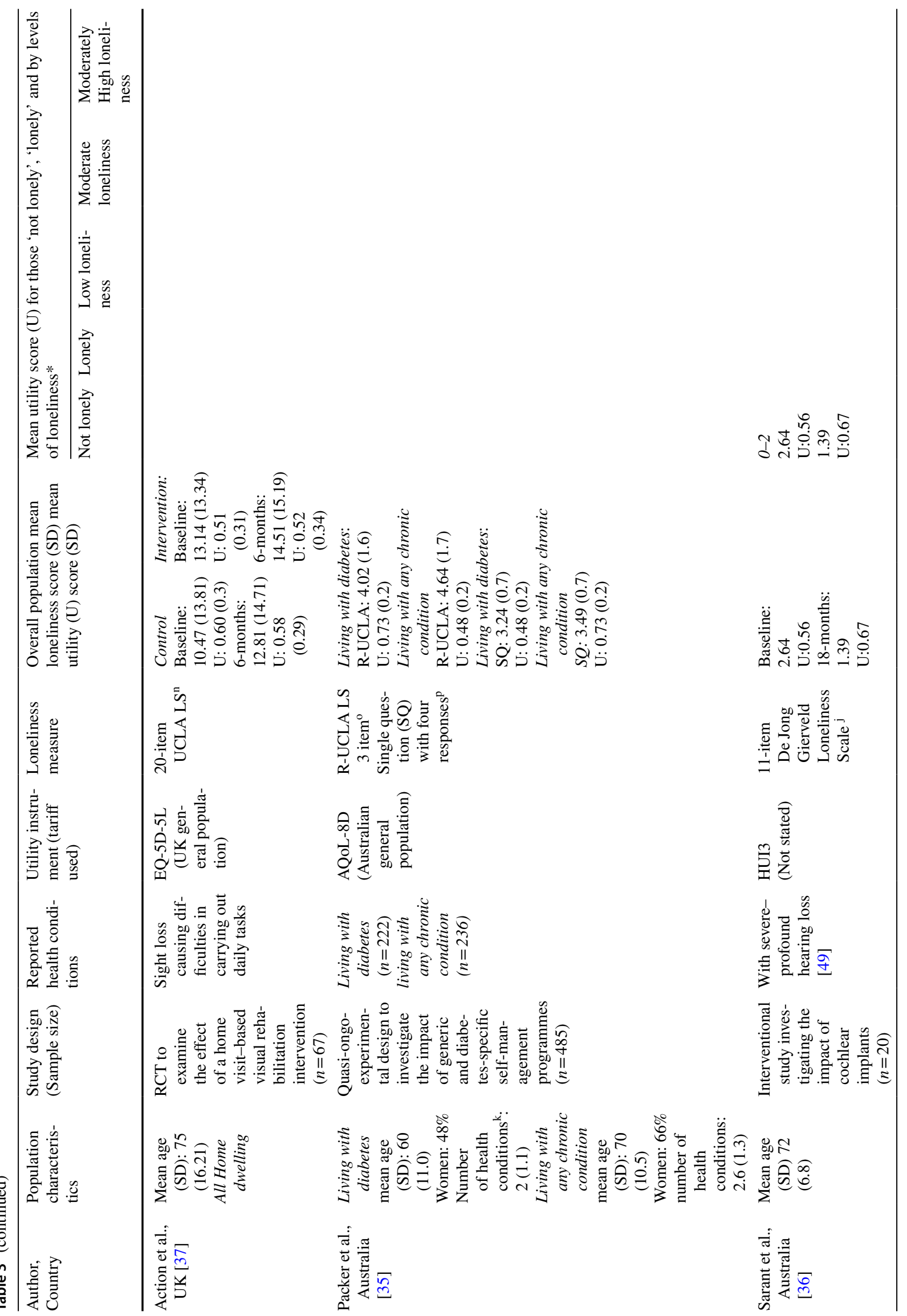



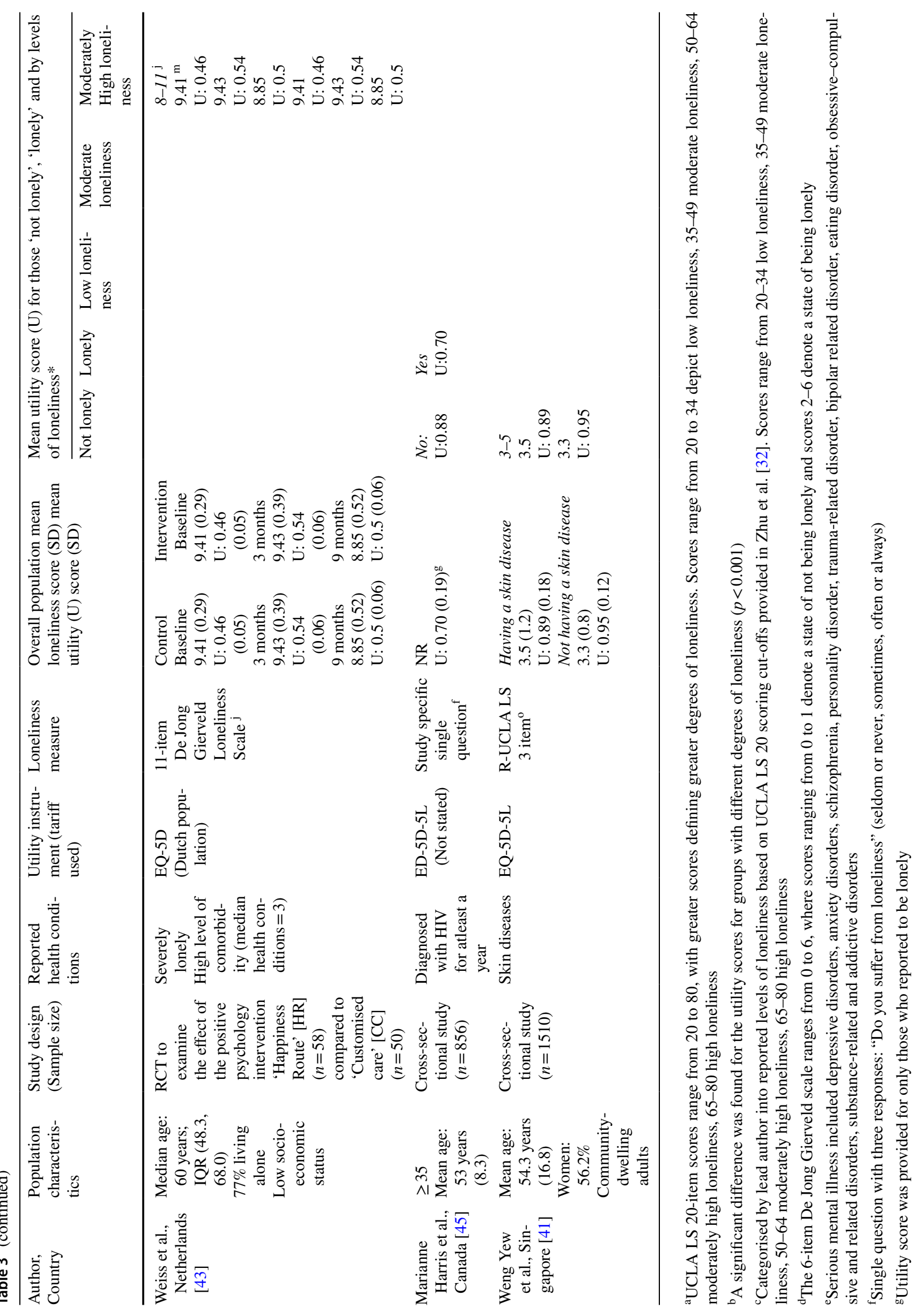


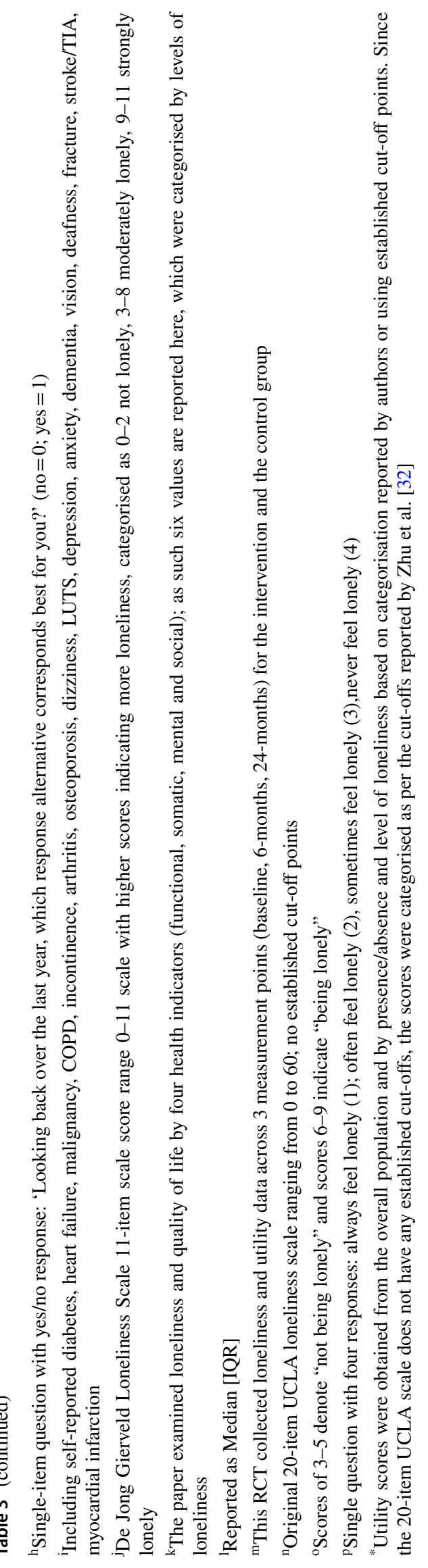

筜 Springer 


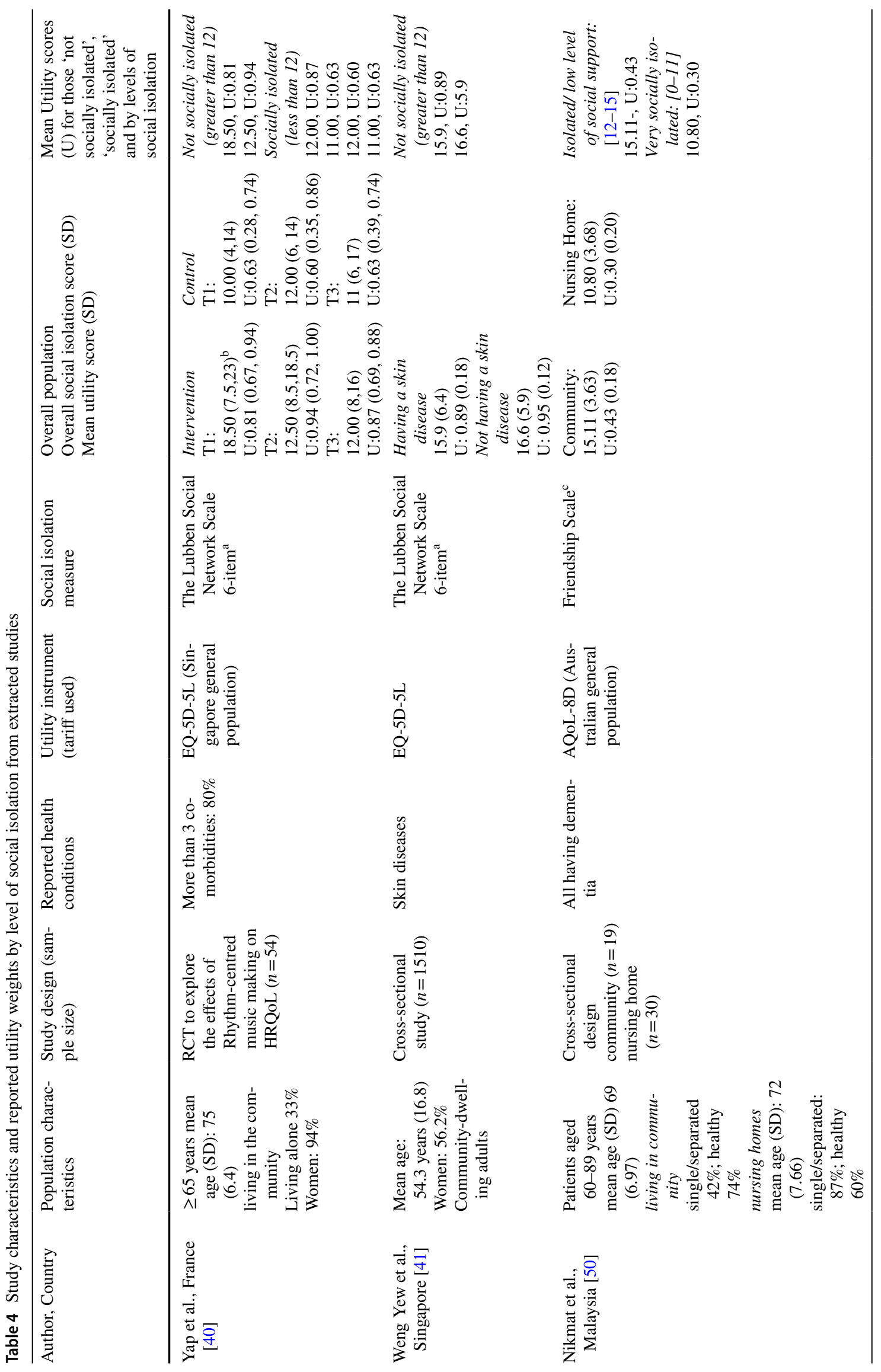




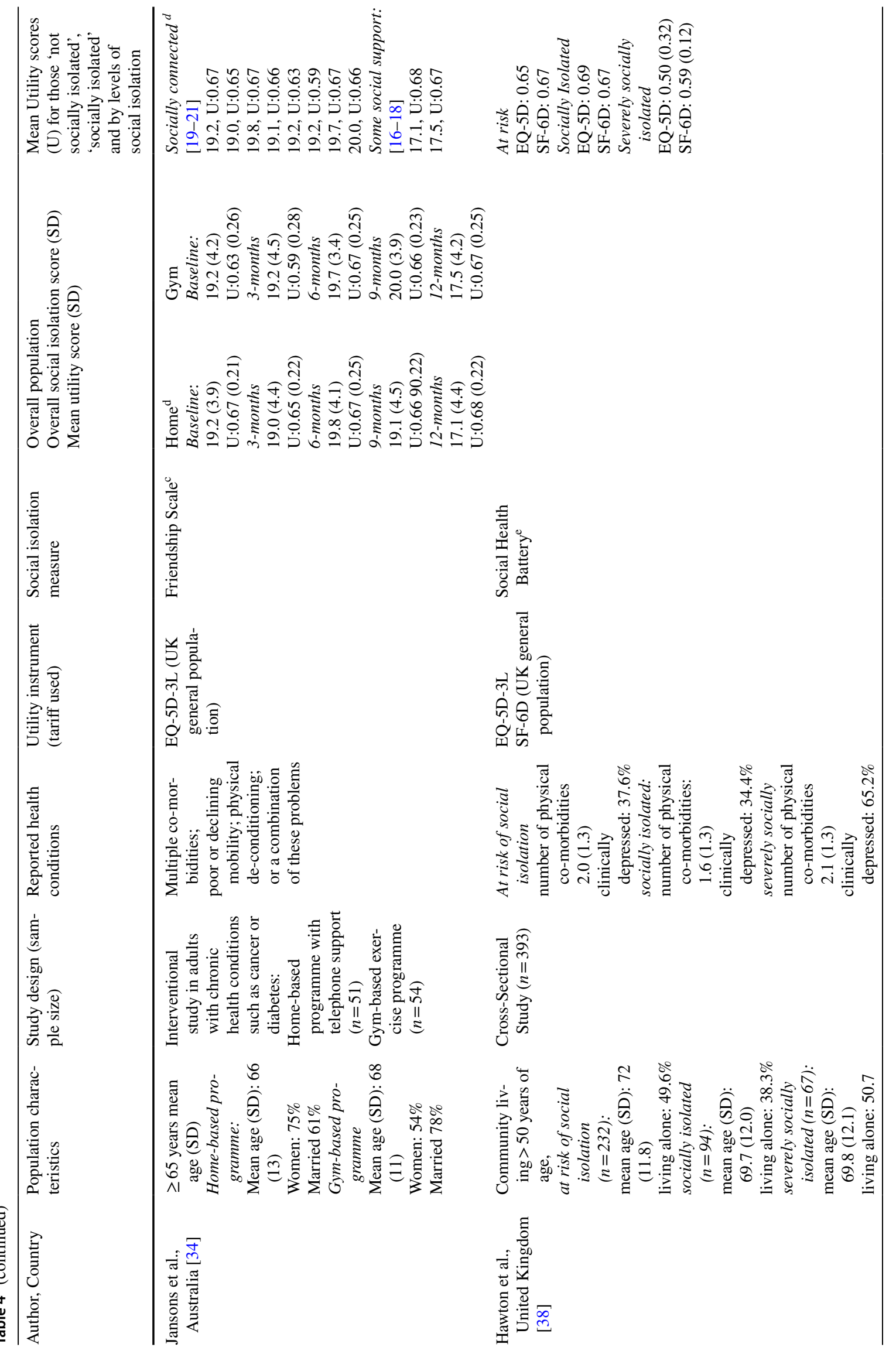




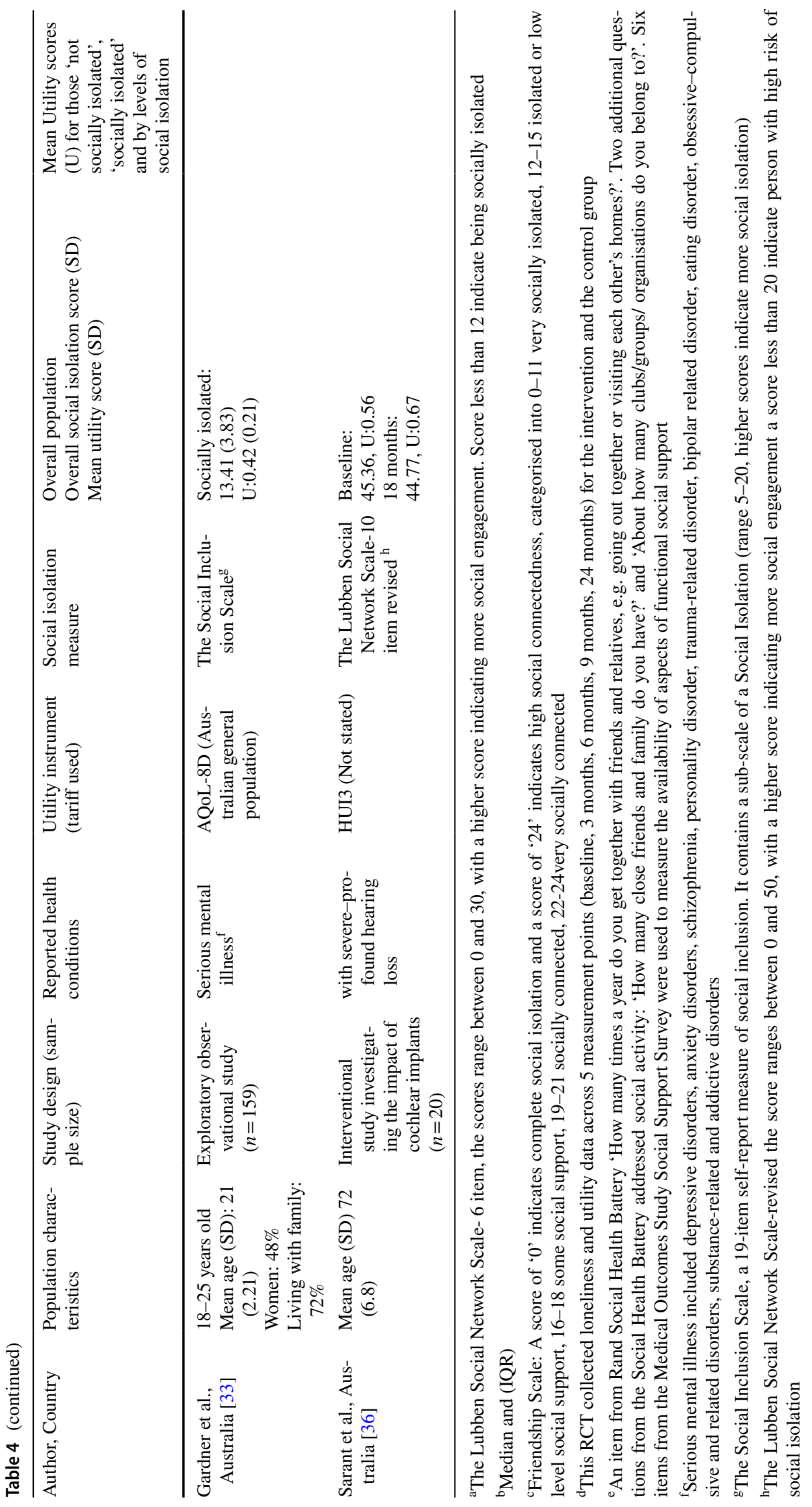


Fig. 2 Reported Utility values across levels of loneliness based on the employed MAUIs

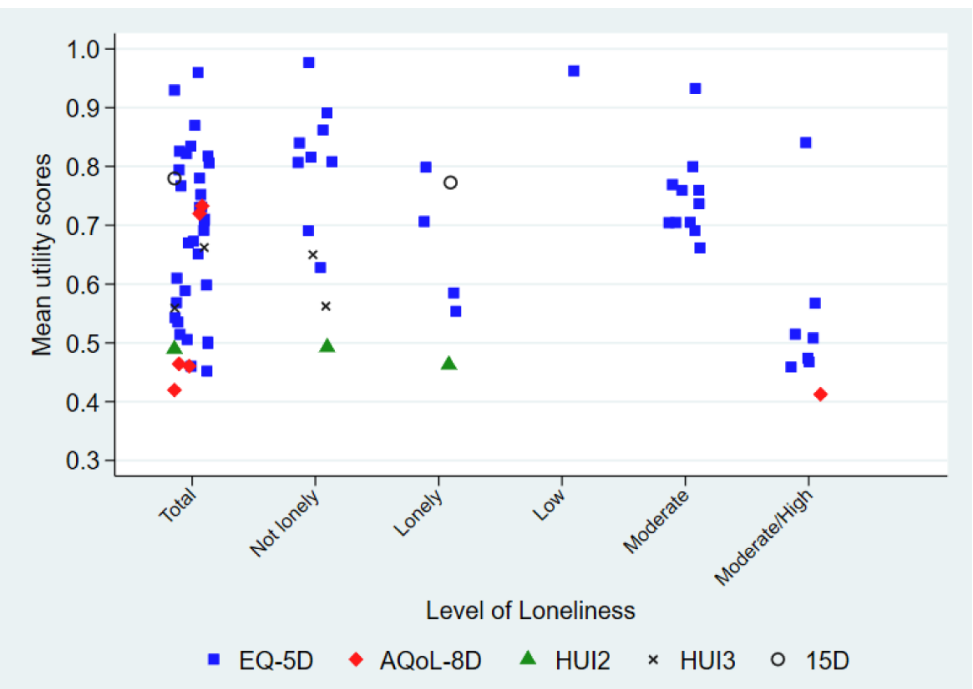

Footnote: The above figure depicts the range of HSUVs across the total population included in the review and provides HSUVs across the presence/absence and severity of loneliness observed across studies. These values are classified based on the MAUls used to examine the utilities. those 'feeling lonely' of 0.46, using the HUI2 [44] and 0.56 using EQ-5D-3L [49] which were lower than the HSUVs of those 'not feeling lonely' 0.50 [44] and 0.60 [49]). Two studies explored HSUVs in individuals with sight loss [37], HIV [45], skin disease [41] and severe-profound hearing loss [36], with HSUVs ranging from 0.51 to 0.89 using the HUI3 and EQ-5D-5L.

A study by Rodriguez-Blazquez et al. explored wellbeing among institutionalised and non-institutionalised older adults, showing that both groups were 'feeling lonely', with those institutionalised having a greater loneliness score as well as a lower HSUVs of 0.57 vs 0.81 in those non-institutionalised measured by the EQ-5D-3L [47]. There was one study that did not report any health conditions in study participants that were classified as 'moderately lonely' with HSUVs ranging from 0.71 to 0.77 , using EQ-5D-3L [39].

Ideally, to generate more precise estimates of the HSUVs of lonely/socially isolated individuals, HSUVs could be pooled together using meta-analysis, thereby providing an average effect size. However, given the limited number of studies, differences in the utility valuation techniques, scoring algorithms, country tariffs and health conditions across study populations, which all lead to high heterogeneity, a quantitative meta-analysis was not possible [51].

\section{Studies measuring social isolation}

Table 4 describes the study characteristics and reported utility weights of six studies measuring social isolation. HSUVs were measured using the EQ-5D-5L [40, 41], EQ-5D-3L [34, 38], AQoL-8D [33, 50], SF-6D [38] and HUI3 [36]. The various instruments used to measure social isolation included the Lubben Social Network scale (LSNS) 6-item [40, 41], Revised-Lubben Social Network scale [36], Friendship scale [34, 50], Social Health Battery [38] and Social inclusion scale [33]. HSUVs by levels of social isolation could not be reported as these measures were not consistently reported across studies.

HSUVs for those reported being socially isolated ranged from 0.3 [50] to 0.87 [40]. A study which explored severity of social isolation in participants with physical co-morbidities as well as depression, reported that those who were severely socially isolated had lower HSUVs of 0.50 and 0.59 measured by the EQ-5D-3L and SF-6D, respectively, compared to the HSUVs of 0.65 and 0.67 in those who reported to be at risk of social isolation [38]. Two other studies included participants with physical co-morbidities [34, 40]. The first study reported HSUVs ranging from 0.63 to 0.87 in those who were socially isolated and 0.81 to 0.94 in those who were not socially isolated [40]. In the second study, the participants reported being socially connected with HSUVs ranging from 0.59 to 0.68 [34]. One study assessed social isolation in participants aged 60 years and above experiencing dementia based on their living arrangements. The participants living in nursing homes reported to be 'very socially isolated' with HSUV of 0.30 which is lower when compared to the participants living in a community setting reporting to be 'isolated' with HSUV of 0.43 [50]. This review also included an article, which explored social isolation in younger adults with serious mental illness reporting a HSUV of 0.43 [33]. 


\section{Discussion}

The aim of this study was to provide an overview of the current state of evidence for the impact of loneliness and/ or social isolation on HSUVs across all age groups, establishing a better understanding of the associated disease burden. Compared to the UK EQ-5D population norms of 0.852 [52] and the Australian AQoL-8D population norms of 0.81 [53], HSUVs associated with loneliness and/or social isolation identified by this review were lower. This suggests that participants experiencing loneliness and/ or social isolation have lower quality of life compared to the general population. Moreover, the lowest HSUV reported for those with a serious mental illness experiencing loneliness and social isolation was 0.42 as measured by the AQoL-8D [33], which is even lower compared to the mean HSUV of 0.67 associated with high prevalence mental disorders in Australia reported using the AQoL-4D [54]. However, these low values may not reflect the true burden of loneliness and social isolation, as HSUVs may have been influenced by many underlying factors, such as the health conditions of the participants, variations in the methods used for the assessment of HSUVs as well as the measurement of loneliness and/ or social isolation. Therefore, the derived HSUVs need to be interpreted carefully, considering many confounding factors that are outlined in the following discussion.

\section{Different measurement tools used to assess loneliness/social isolation}

The UCLA loneliness scale and the De Jong Gierveld loneliness scale were the most frequently used measures for assessing loneliness, whereas the Lubben Social Network scale and Friendship scale were most often used for measuring social isolation. However, we found variations in the use of terminology and concepts of loneliness and social isolation and the corresponding use of measures. For instance, a study that claimed to measure social isolation used the R-UCLA, which is a measure of subjective loneliness [35]. The De Jong Gierveld Loneliness scale differentiates between two types of loneliness, including social loneliness and emotional loneliness. Only one of the 5 studies which used this instrument to measure loneliness reported scores within the two dimensions [39]. As our review focused only on overall loneliness scores, further studies could explore HSUVs associated with both types, social and emotional loneliness. Some included studies categorised levels of social isolation referring to concepts such as 'social support' or 'social connectedness' $[34,50]$, which may not be reliable indicators of social isolation [55] demonstrating lack of conceptual clarity around these terms. While the above stated instruments are validated measures [56-59], we also found many studies that used study specific single item questions to identify people experiencing loneliness. While it is problematic to use non-validated measures, it is also known that such direct measurement techniques, generally result in underreporting of loneliness due to the associated social stigma attached to loneliness [60].

\section{Difference across levels of loneliness/social isolation}

Among those studies which included participants with a subjective feeling of loneliness [32, 33, 48, 49], one study reported HSUVs by the degree of loneliness [32]. This study reported decreasing HSUVs by increasing levels of loneliness as measured by the EQ-5D-3L. Two studies by Taube et al. and Maxwell et al. reported HSUVs of those lonely vs no lonely and found that the HSUVs were lower in the lonely group than those not feeling lonely [44, 49]. Another study, which categorised HSUVs based on levels of social isolation, reported lower HSUVs in the severely socially isolated group compared to those at a lower risk of social isolation [38]. Similar observations were noticed in a study by Nikmat et al. which reported a lower HSUV in those who were very socially isolated compared to those who had lower levels of social support. [50]. However, in all the above studies, the participants reported co-morbidities, which makes it challenging to extract the excess health burden associated with loneliness.

While the focus of this review was on both loneliness and social isolation, it remains unclear whether there is a greater health burden, in terms of reductions in HSUV, associated with loneliness and/or social isolation. Gardner et al. and Sarant et al. were the only two studies that included measures of both loneliness and social isolation $[33,36]$, although the later study sample neither experienced loneliness nor social isolation. Participants in the Gardner et al. study consisting of young adults with serious mental illness, reported higher levels of loneliness while not being socially isolated with a HSUV of 0.42 . As this study did not adjust for health conditions, whether the lower HSUV reflects the burden of loneliness cannot be clearly confirmed. Although all studies have included participants with pre-existing health conditions, it would be of interest to further explore HSUVs in those experiencing loneliness/social isolation without any underlying health conditions.

\section{Different MAUls used across studies}

It has been previously shown that different MAUIs produce different HSUVs [61] and it was observed that there were several MAUIs used to assess HSUVs across the included 
studies. The impact of the choice of MAUI was observed in the study by Hawton et al. (2010), where different HSUVs across different levels of social isolation were reported when using the EQ-5D-3L compared with the SF-6D [38]. These differences are likely due to the conceptual differences between MAUIs. For example, the EQ-5D-3L, used in most of the studies to derive HSUVs, mainly focuses on physical health [62] but does not contain any items related to social functioning. Contrastingly, the SF-6D contains equal item in both physical and psychosocial domains. Similarly, the AQoL-8D includes items related to social isolation, social exclusion and satisfaction with close relationships, which could potentially capture the feeling of loneliness/social isolation. In our review, the lowest HSUV of 0.42 for participants classified as moderately lonely' [33] and the lowest HSUV of 0.30 for those reporting as being highly socially isolated [50], was measured using the AQoL-8D. This suggests that the AQoL-8D may be more sensitive in capturing aspects of loneliness/social isolation. However, further research is needed to validate this observation as the studies included patient with serious mental illness [33] and chronic conditions [35] and thus, it cannot be ruled out whether decrements in HSUVs were solely driven by loneliness.

\section{Presence of multi-morbidities}

Most of the studies included older adults with various physical and mental health conditions, which means that the HSUVs observed could have been driven by the presence of existing multi-morbidities. Previous studies have reported reductions in HSUVs associated with health conditions such as serious mental illness [61]. As loneliness and social isolation have negative impacts on the physical and psychological health of older people leading to serious health consequences [63], it is difficult to understand the excess burden attributable to loneliness and/or social isolation. There were studies that included specific health conditions, such as sight loss [37], diabetes [35], serious mental illness [33] and profound hearing loss [36]. Therefore, to inform future modelling studies, there will be a need for estimates of the joined health condition HSUV, considering both co-morbidities and levels of loneliness [64].

\section{Difference among community-dwelling and institutionalised populations}

The living situation of study participants could also affect HSUVs reported in this review. Only two studies compared HSVUs between older people living in residential aged care and community settings $[47,50]$. Both studies reported a lower HSUV in those institutionalised, who also reported higher levels of loneliness [47] and social isolation [50]. This is consistent with previous literature, which has stated that loneliness is higher in those living in institutions compared to community-dwelling older adults [56, 65]. However, it is important to consider the various confounding aspects to extract out the true burden on loneliness and/or social isolation.

\section{Limitations and further research}

The current paucity of published utility weights by severity status of loneliness and social isolation is reflected in the lack of cost-utility analysis of loneliness interventions. This is the first systematic review that has explored HSUVs associated with loneliness and social isolation using a broad range of inclusion criteria. The HSUVs identified could serve as input parameters for future modelled economic evaluations, although the uncertainty within the body of literature needs to be considered for appropriate use of HSUVs. Our study has certain limitations and results should be interpreted with caution. First, we used cut-off points reported by Zhu et al. to define levels of loneliness [32]. It is possible that different categorisation of cut-off points may have resulted in different corresponding HSUVs. Second, due to heterogeneity of the studies, we could not conduct a meta-analysis to synthesise estimates of health utilities [66]. Third, a small number of studies were included in this review, which underscores the existing research gap and the need for future studies. Due to lack of standardised checklists for assessing the quality of HSUVs, we did not conduct quality assessments of the included studies. The arrival of the Covid-19 pandemic and its impact on the burden of loneliness and social isolation has been a growing concern. Although we re-ran the search at the beginning of 2021, no studies exploring the impact of Covid-19 had been published which met the criteria for inclusion in the review. We may expect more studies to arise, incorporating the additional burden on loneliness and social isolation due to the Covid-19 pandemic.

\section{Conclusion}

This study is the first systematic review providing summary of existing HSUVs by severity of loneliness and/or social isolation across age groups, demonstrating the paucity of evidence available around specific health burden of loneliness and/or social isolation. With a broad range of reported HSUVs, this review highlights the challenges in identifying reliable estimates of HSUVs that are essential input parameters in model-based economic evaluations of interventions targeted to address loneliness and /or social isolation. These challenges mainly arise due to (i) differences in the scoring algorithms and descriptive systems of MAUIs that result in different weights in deriving the HSUVs, (ii) differences in the measurement of loneliness and social isolation, (iii) and 
the confounding effects of health conditions of the respondents and different settings. Despite these observed challenges, this review reflects detriments in utility which are important indicators of the burden associated with loneliness and social isolation. Further studies exploring HSUVs across levels of loneliness and social isolation in a systematic and justified method are encouraged.

Supplementary Information The online version contains supplementary material available at https://doi.org/10.1007/s11136-021-03063-1.

Funding Open Access funding enabled and organized by CAUL and its Member Institutions.

Open Access This article is licensed under a Creative Commons Attribution 4.0 International License, which permits use, sharing, adaptation, distribution and reproduction in any medium or format, as long as you give appropriate credit to the original author(s) and the source, provide a link to the Creative Commons licence, and indicate if changes were made. The images or other third party material in this article are included in the article's Creative Commons licence, unless indicated otherwise in a credit line to the material. If material is not included in the article's Creative Commons licence and your intended use is not permitted by statutory regulation or exceeds the permitted use, you will need to obtain permission directly from the copyright holder. To view a copy of this licence, visit http://creativecommons.org/licenses/by/4.0/.

\section{References}

1. Loneliness: A new public health challenge. (2019). Retrieved April 1, 2020, from https://www.vichealth.vic.gov.au/letter/artic les/vh-letter-47-loneliness

2. Victor, C. R., \& Yang, K. (2012). The prevalence of loneliness among adults: A case study of the United Kingdom. Journal of Psychology, 146(1-2), 85-104. https://doi.org/10.1080/00223980. 2011.613875

3. Peplau, L., \& Perlman, D. (1982). Loneliness: A sourcebook of current theory, research and therapy. Wiley.

4. Routasalo, P. E., Savikko, N., Tilvis, R. S., Strandberg, T. E., \& Pitkala, K. H. (2006). Social contacts and their relationship to loneliness among aged people-A population-based study. Gerontology, 52(3), 181-187. https://doi.org/10.1159/000091828

5. Cornwell, E. Y., \& Waite, L. J. (2009). Social disconnectedness, perceived isolation, and health among older adults. Journal of Health and Social Behavior, 50(1), 31-48. https://doi.org/10. 1177/002214650905000103

6. Durcan, D., \& Bell, R. (2015). Local action on health inequalities: reducing social isolation across the lifecourse. Public Health England.

7. Gardiner, C., Geldenhuys, G., \& Gott, M. (2018). Interventions to reduce social isolation and loneliness among older people: An integrative review. Health and Social Care in the Community, 26(2), 147-157. https://doi.org/10.1111/hsc.12367

8. Knox, S. S., \& Uvnas-Moberg, K. (1998). Social isolation and cardiovascular disease: An atherosclerotic pathway? Psychoneuroendocrinology, 23(8), 877-890.

9. James, B. D., Wilson, R. S., Barnes, L. L., \& Bennett, D. A. (2011). Late-life social activity and cognitive decline in old age. Journal of the International Neuropsychological Society, 17(6), 998-1005. https://doi.org/10.1017/S1355617711000531
10. Hawkley, L. C., Thisted, R. A., Masi, C. M., \& Cacioppo, J. T. (2010). Loneliness predicts increased blood pressure: 5 -year cross-lagged analyses in middle-aged and older adults. Psychology and Aging, 25(1), 132-141. https://doi.org/10.1037/a0017805

11 Cohen, S., Doyle, W. J., Skoner, D. P., Rabin, B. S., \& Gwaltney, J. M., Jr. (1997). Social ties and susceptibility to the common cold. JAMA, 277(24), 1940-1944.

12. Steptoe, A., Shankar, A., Demakakos, P., \& Wardle, J. (2013). Social isolation, loneliness, and all-cause mortality in older men and women. Proceedings of the National Academy of Sciences of the United States of America, 110(15), 5797-5801. https://doi.org/ 10.1073/pnas.1219686110

13. Kuiper, J. S., Zuidersma, M., Oude Voshaar, R. C., Zuidema, S. U., van den Heuvel, E. R., Stolk, R. P., et al. (2015). Social relationships and risk of dementia: A systematic review and metaanalysis of longitudinal cohort studies. Ageing Research Reviews, 22, 39-57. https://doi.org/10.1016/j.arr.2015.04.006

14. Cacioppo, J. T., Hughes, M. E., Waite, L. J., Hawkley, L. C., \& Thisted, R. A. (2006). Loneliness as a specific risk factor for depressive symptoms: Cross-sectional and longitudinal analyses. Psychology and Aging, 21(1), 140-151. https://doi.org/10. 1037/0882-7974.21.1.140

15. Holt-Lunstad, J., Smith, T. B., Baker, M., Harris, T., \& Stephenson, D. (2015). Loneliness and social isolation as risk factors for mortality: A meta-analytic review. Perspectives on Psychological Science, 10(2), 227-237. https://doi.org/10.1177/17456 91614568352

16. Perissinotto, C. M., StijacicCenzer, I., \& Covinsky, K. E. (2012). Loneliness in older persons: A predictor of functional decline and death. Archives of Internal Medicine, 172(14), 1078-1083. https://doi.org/10.1001/archinternmed.2012.1993

17. Cacioppo, S., Grippo, A. J., London, S., Goossens, L., \& Cacioppo, J. T. (2015). Loneliness: Clinical import and interventions. Perspectives on Psychological Science, 10(2), 238249. https://doi.org/10.1177/1745691615570616

18. Cohen-Mansfield, J., \& Perach, R. (2015). Interventions for alleviating loneliness among older persons: A critical review. American Journal of Health Promotion, 29(3), e109-125. https://doi. org/10.4278/ajhp.130418-LIT-182

19. Masi, C. M., Chen, H. Y., Hawkley, L. C., \& Cacioppo, J. T. (2011). A meta-analysis of interventions to reduce loneliness. Personality and Social Psychology Review, 15(3), 219-266. https://doi.org/10.1177/1088868310377394

20. Poscia, A., Stojanovic, J., La Milia, D. I., Duplaga, M., Grysztar, M., Moscato, U., et al. (2018). Interventions targeting loneliness and social isolation among the older people: An update systematic review. Experimental Gerontology, 102, 133-144. https://doi.org/10.1016/j.exger.2017.11.017

21. Drummond, M. F., Claxton, K., Sculpher, M. J., Stoddart, G. L., \& Torrance, G. W. (2015). Methods for the economic evaluation of health care programmes (4th ed.). Oxford University Press.

22. Richardson, J., McKie, J., \& Bariola, E. (2014). Multiattribute utility instruments and their use. Encyclopedia of Health Economics, 2, 341-357.

23. Brooks, R., Boye, K. S., \& Slaap, B. (2020). EQ-5D: A plea for accurate nomenclature. J Patient Rep Outcomes, 4(1), 52. https://doi.org/10.1186/s41687-020-00222-9

24. Richardson, J., Khan, M. A., Iezzi, A., \& Maxwell, A. (2015). Comparing and explaining differences in the magnitude, content, and sensitivity of utilities predicted by the EQ-5D, SF-6D, HUI 3, 15D, QWB, and AQoL-8D multiattribute utility instruments. Medical Decision Making, 35(3), 276-291. https://doi. org/10.1177/0272989X14543107

25. Beaudet, A., Clegg, J., Thuresson, P. O., Lloyd, A., \& McEwan, P. (2014). Review of utility values for economic modeling in 
type 2 diabetes. Value Health, 17(4), 462-470. https://doi.org/ 10.1016/j.jval.2014.03.003

26. Brown, J., Cook, K., Adamski, K., Lau, J., Bargo, D., Breen, S., et al. (2017). Utility values associated with advanced or metastatic non-small cell lung cancer: Data needs for economic modeling. Expert Review of Pharmacoeconomics \&amp; Outcomes Research, 17(2), 153-164. https://doi.org/10.1080/14737 167.2017.1311210

27. Sonntag, M., Konig, H. H., \& Konnopka, A. (2013). The estimation of utility weights in cost-utility analysis for mental disorders: A systematic review. PharmacoEconomics, 31(12), 1131-1154. https://doi.org/10.1007/s40273-013-0107-9

28. Brown, V., Tan, E. J., Hayes, A. J., Petrou, S., \& Moodie, M. L. (2018). Utility values for childhood obesity interventions: A systematic review and meta-analysis of the evidence for use in economic evaluation. Obesity Reviews, 19(7), 905-916. https:// doi.org/10.1111/obr.12672

29. Paracha, N., Abdulla, A., \& MacGilchrist, K. S. (2018). Systematic review of health state utility values in metastatic non-small cell lung cancer with a focus on previously treated patients. Health and Quality of Life Outcomes, 16(1), 179. https://doi.org/10.1186/ s12955-018-0994-8

30. Moher, D., Liberati, A., Tetzlaff, J., Altman, D. G., Group P. (2009). Preferred reporting items for systematic reviews and metaanalyses: The PRISMA statement. $B M J, 339$, b2535. https://doi. org/10.1136/bmj.b2535

31 MouradOuzzani, H. H., Fedorowicz, Z., \& Elmagarmid, A. (2016). Rayyan-A web and mobile app for systematic reviews. Systematic Reviews. https://doi.org/10.1186/s13643-016-0384-4

32. Zhu, Y., Liu, J., Qu, B., \& Yi, Z. (2018). Quality of life, loneliness and health-related characteristics among older people in Liaoning province, China: A cross-sectional study. British Medical Journal Open, 8(11), e021822. https://doi.org/10.1136/bmjop en-2018-021822

33. Gardner, A., Cotton, S., O’Donoghue, B., Killackey, E., Norton, P. J., \& Filia, K. (2020). Preliminary psychometric properties of a measure of social inclusion for young adults aged 18 to 25 with serious mental illness. Psychiatric Rehabilitation Journal, 43(2), 156-169. https://doi.org/10.1037/prj0000382

34. Jansons, P., Robins, L., O'Brien, L., \& Haines, T. (2017). Gymbased exercise and home-based exercise with telephone support have similar outcomes when used as maintenance programs in adults with chronic health conditions: A randomised trial. Journal of Physiotherapy, 63(3), 154-160. https://doi.org/10.1016/j.jphys. 2017.05.018

35. Packer, T. L., Boldy, D., Ghahari, S., Melling, L., Parsons, R., \& Osborne, R. H. (2012). Self-management programs conducted within a practice setting: Who participates, who benefits and what can be learned? Patient Education and Counseling, 87(1), 93-100. https://doi.org/10.1016/j.pec.2011.09.007

36. Sarant, J., Harris, D., Busby, P., Maruff, P., Schembri, A., Dowell, R., et al. (2019). The effect of cochlear implants on cognitive function in older adults: Initial baseline and 18-month follow up results for a prospective international longitudinal study. Frontiers in Neuroscience, 13, 789. https://doi.org/10.3389/fnins.2019. 00789

37. Acton, J. H., Molik, B., Court, H., \& Margrain, T. H. (2016). Effect of a home visit-based low vision rehabilitation intervention on visual function outcomes: An exploratory randomized controlled trial. Investigative Ophthalmology \&amp; Visual Science, 57(15), 6662-6667. https://doi.org/10.1167/iovs.16-19901

38. Hawton, A., Green, C., Dickens, A. P., Richards, S. H., Taylor, R. S., Edwards, R., et al. (2011). The impact of social isolation on the health status and health-related quality of life of older people. Quality of Life Research, 20(1), 57-67. https://doi.org/10.1007/ s11136-010-9717-2
39. Mountain, G., Windle, G., Hind, D., Walters, S., Keertharuth, A., Chatters, R., et al. (2017). A preventative lifestyle intervention for older adults (lifestyle matters): A randomised controlled trial. Age and Ageing, 46(4), 627-634. https://doi.org/10.1093/ageing/ afx021

40. Yap, A. F., Kwan, Y. H., Tan, C. S., Ibrahim, S., \& Ang, S. B. (2017). Rhythm-centred music making in community living elderly: A randomized pilot study. BMC Complementary and Alternative Medicine, 17(1), 311. https://doi.org/10.1186/ s12906-017-1825-x

41. Yew, Y. W., Kuan, A. H. Y., Ge, L., Yap, C. W., \& Heng, B. H. (2020). Psychosocial impact of skin diseases: A population-based study. PLoS ONE, 15(12), e0244765. https://doi.org/10.1371/ journal.pone. 0244765

42. van Houwelingen, A. H., den Elzen, W. P., le Cessie, S., Blom, J. W., \& Gussekloo, J. (2015). Consequences of interaction of functional, somatic, mental and social problems in communitydwelling older people. PLoS ONE, 10(4), e0121013. https://doi. org/10.1371/journal.pone.0121013

43. Weiss, L. A., Oude Voshaar, M. A. H., Bohlmeijer, E. T., \& Westerhof, G. J. (2020). The long and winding road to happiness: A randomized controlled trial and cost-effectiveness analysis of a positive psychology intervention for lonely people with health problems and a low socio-economic status. Health and Quality of Life Outcomes, 18(1), 162. https://doi.org/10. 1186/s12955-020-01416-x

44. Maxwell, C. J., Kang, J., Walker, J. D., Zhang, J. X., Hogan, D. B., Feeny, D. H., et al. (2009). Sex differences in the relative contribution of social and clinical factors to the Health Utilities Index Mark 2 measure of health-related quality of life in older home care clients. Health and Quality of Life Outcomes, 7, 80. https://doi.org/10.1186/1477-7525-7-80

45. Harris, M., Brouillette, M. J., Scott, S. C., Smaill, F., Smith, G., Thomas, R., et al. (2020). Impact of loneliness on brain health and quality of life among adults living with HIV in Canada. Journal of Acquired Immune Deficiency Syndromes, 84(4), 336-344. https://doi.org/10.1097/QAI.0000000000002355

46. Ko, H., Park, Y. H., Cho, B., Lim, K. C., Chang, S. J., Yi, Y. M., et al. (2019). Gender differences in health status, quality of life, and community service needs of older adults living alone. Archives of Gerontology and Geriatrics, 83, 239-245. https:// doi.org/10.1016/j.archger.2019.05.009

47. Rodriguez-Blazquez, C., Forjaz, M. J., Prieto-Flores, M. E., Rojo-Perez, F., Fernandez-Mayoralas, G., Martinez-Martin, P., et al. (2012). Health status and well-being of older adults living in the community and in residential care settings: Are differences influenced by age? Aging \&amp; Mental Health, 16(7), 884-891. https://doi.org/10.1080/13607863.2012.684664

48. Liira, H., Mavaddat, N., Eineluoto, M., Kautiainen, H., Strandberg, T., Suominen, M., et al. (2018). Health-related quality of life as a predictor of mortality in heterogeneous samples of older adults. European Geriatric Medicine, 9(2), 227-234. https://doi.org/10.1007/s41999-018-0029-3

49. Taube, E., Kristensson, J., Sandberg, M., Midlov, P., \& Jakobsson, U. (2015). Loneliness and health care consumption among older people. Scandinavian Journal of Caring Sciences, 29(3), 435-443. https://doi.org/10.1111/scs.12147

50. Nikmat, A. W., Hawthorne, G., \& Al-Mashoor, S. H. (2015). The comparison of quality of life among people with mild dementia in nursing home and home care-a preliminary report. Dementia (London), 14(1), 114-125. https://doi.org/10.1177/ 1471301213494509

51. Peasgood, T., \& Brazier, J. (2015). Is meta-analysis for utility values appropriate given the potential impact different elicitation methods have on values? PharmacoEconomics, 33(11), 1101-1105. https://doi.org/10.1007/s40273-015-0310-y 
52. Szende, A., Janssen, B., \& Cabases, J. (2014). Self-reported population health: An International perspective based on $E Q-5 D$. Springer.

53. Hawthorne, G., Korn, S., \& Richardson, J. (2013). Population norms for the AQoL derived from the 2007 Australian National Survey of Mental Health and Wellbeing. Australian and New Zealand Journal of Public Health, 37(1), 7-16. https://doi.org/ 10.1111/1753-6405.12004

54. Mihalopoulos, C., Engel, L., Le, L. K., Magnus, A., Harris, M., \& Chatterton, M. L. (2018). Health state utility values of high prevalence mental disorders in Australia: Results from the National Survey of Mental Health and Wellbeing. Quality of Life Research, 27(7), 1815-1825. https://doi.org/10.1007/ s11136-018-1843-2

55. Valtorta, N. K., Kanaan, M., Gilbody, S., \& Hanratty, B. (2016). Loneliness, social isolation and social relationships: What are we measuring? A novel framework for classifying and comparing tools. British Medical Journal Open, 6(4), e010799. https://doi. org/10.1136/bmjopen-2015-010799

56. Hawthorne, G. (2006). Measuring social isolation in older adults: Development and initial validation of the friendship Scale. Social Indicators Research, 77(3), 521-548. https://doi.org/10.1007/ s11205-005-7746-y

57. Myagmarjav, S., Burnette, D., \& Goeddeke, F., Jr. (2019). Comparison of the 18-item and 6-item Lubben Social Network Scales with community-dwelling older adults in Mongolia. PLoS ONE, 14(4), e0215523. https://doi.org/10.1371/journal.pone.0215523

58. Robustelli, B. L., Newberry, R. E., Whisman, M. A., \& Mittal, V. A. (2017). Social relationships in young adults at ultra high risk for psychosis. Psychiatry Research, 247, 345-351. https://doi.org/ 10.1016/j.psychres.2016.12.008

59 Gierveld, J. D. J., \& Tilburg, T. V. (2006). A 6-item scale for overall, emotional, and social loneliness confirmatory tests on survey data. Research on Aging, 28(5), 582-598.

60. Nicolaisen, M., \& Thorsen, K. (2014). Who are lonely? Loneliness in different age groups (18-81 years old), using two measures of loneliness. International Journal of Aging and Human Development, 78(3), 229-257. https://doi.org/10.2190/AG.78.3.b
61. Mihalopoulos, C., Chen, G., Iezzi, A., Khan, M. A., \& Richardson, J. (2014). Assessing outcomes for cost-utility analysis in depression: Comparison of five multi-attribute utility instruments with two depression-specific outcome measures. British Journal of Psychiatry, 205(5), 390-397. https://doi.org/10.1192/bjp.bp. 113.136036

62. Reed, C., Barrett, A., Lebrec, J., Dodel, R., Jones, R. W., Vellas, B., et al. (2017). How useful is the EQ-5D in assessing the impact of caring for people with Alzheimer's disease? Health and Quality of Life Outcomes, 15(1), 16. https://doi.org/10.1186/ s12955-017-0591-2

63. Chen, Y., Hicks, A., \& While, A. E. (2014). Quality of life and related factors: A questionnaire survey of older people living alone in Mainland China. Quality of Life Research, 23(5), 15931602. https://doi.org/10.1007/s11136-013-0587-2

64. Thompson, A. J., Sutton, M., \& Payne, K. (2019). Estimating joint health condition utility values. Value Health, 22(4), 482-490. https://doi.org/10.1016/j.jval.2018.09.2843

65. Winningham, R. G., \& Pike, N. L. (2007). A cognitive intervention to enhance institutionalized older adults' social support networks and decrease loneliness. Aging \&amp; Mental Health, 11(6), 716-721. https://doi.org/10.1080/13607860701366228

66. Petrou, S., Kwon, J., \& Madan, J. (2018). A practical guide to conducting a systematic review and meta-analysis of health state utility values. PharmacoEconomics, 36(9), 1043-1061. https:// doi.org/10.1007/s40273-018-0670-1

67. Pitkala, K. H., Routasalo, P., Kautiainen, H., \& Tilvis, R. S. (2009). Effects of psychosocial group rehabilitation on health, use of health care services, and mortality of older persons suffering from loneliness: A randomized, controlled trial. Journals of Gerontology. Series A, Biological Sciences and Medical Sciences, 64(7), 792-800. https://doi.org/10.1093/gerona/glp011

Publisher's Note Springer Nature remains neutral with regard to jurisdictional claims in published maps and institutional affiliations. 